\title{
ESTILO COGNITIVO Y LOGRO ACADÉMICO EN LA CIUDAD DE BOGOTÁ
}

Christian Hederich M. $^{*}$

Ángela Camargo U. *

\section{El proyecto}

\section{Contexto}

Desde hace ya 10 años, se ha venido acumulando información acerca de los muy escasos niveles de logro educativo de los estudiantes colombianos (MEN-Saber, 1992, 1995; TIMSS, 1997; UN-SED, 1999; entre otras). Sin embargo, a pesar de las precisiones alcanzadas respecto de la descripción de tales niveles, hasta el momento se ha avanzado poco en la explicación de los mismos. De esta manera, los estudios realizados sobre los factores asociados con el logro académico, aunque han arrojado información importante, ésta tiene aún un carácter más bien inicial (DNP, 1999).

A nuestro juicio, buena parte de esta dificultad explicativa radica en la escasa base teórica que caracteriza los estudios clásicos de factores asociados, usualmente realizados desde una óptica economicista, lo cual está en el origen de su bajo poder explicativo habitual. En general, este tipo de estudios, particularmente aquellos que trabajan con grandes muestras, son observaciones puntuales que hacen un recuento de variables asociables, de forma intuitiva, con el logro, sin explicación sobre el modelo teórico en el que se consideran dichas variables. Así, la dispersión de éstas y la ausencia frecuente de una conceptualización clara y consistente acerca de las asociaciones que se esperan encontrar hacen de la lectura de muchos de los estudios de factores asociados con el logro poco más que el recuento de una lista desordenada de características varias. De tal forma, "factores" como el género del estudiante, su nivel socioeconómico, el tamaño de su familia, los niveles de formación docente de uno o varios de sus maestros, la presencia de material didáctico en la escuela, el género de los maestros, la disponibilidad de servicios públicos en la escuela, etc., se incluyen en estos estudios como resultados de intuiciones poco desarrolladas y aún menos explícitas. Esto ha conducido a que una buena porción de las variables incluidas sean factores gruesos, usualmente indicadores amplios, que agrupan multitud de variables más finas, no consideradas específicamente, pero con vínculos conceptuales mucho más claros con el logro académico.

Desde nuestro punto de vista, el problema del logro académico es, en últimas, un problema de aprendizaje en el contexto escolar y, por lo tanto, de naturaleza esencialmente educativa, que debe explicarse en términos psicopedagógicos desde una orientación teórica cognitiva. Así, hemos querido superar las limitaciones de los estudios convencionales sobre factores asociados, tomando como base, un marco teórico de enorme desarrollo en la psicología cognitiva: el de la Teoría de la Diferenciación Psicológica, a partir de la cual surge el concepto de estilo cognitivo. El uso de este "lente teórico" presenta, a nuestro juicio una serie de ventajas importantes para el estudio del logro académico:

\footnotetext{
* Profesores de la Universidad Pedagógica Nacional, Centro de Investigaciones CIUP
} 
- En primer lugar, el estilo cognitivo es una característica individual, de naturaleza psicológica, estrechamente ligada al funcionamiento cognitivo, y por lo tanto, al proceso del aprendizaje. El concepto de estilo cognitivo es intuitivamente simple: es una modalidad de funcionamiento cognitivo que no se refiere al contenido, sino a la forma en la que se da el proceso de aprendizaje. Esto es, salvo el caso de limitaciones excepcionales, cualquier persona de cualquier estilo cognitivo puede aprender y manejar cualquiera de los contenidos escolares; pero, en la medida en que la forma como ocurre el proceso es diferente para cada estilo, no es descabellado esperar una asociación entre el estilo cognitivo y el logro académico, dada por la medida de la pertinencia de la acción pedagógica. Así, cuando asumimos el estilo cognitivo como unidad de aná.

- En segundo lugar, sabemos que el estilo cognitivo surge como resultado de la interacción entre multitud de variables individuales, familiares y culturales. Así, desde un punto de vista teórico, es posible explicar; a partir del estilo cognitivo, la asociación entre un factor y el logro de aprendizaje por la vía de la mediación que éste establece entre el factor considerado y el logro. En la medida en que un factor contribuya a la conformación del estilo cognitivo y en que el estilo se encuentre relacionado con el logro de aprendizaje, hemos explicado la asociación aparente entre el factor y el logro.

- En tercer lugar, están las enormes posibilidades de acción efectiva que ofrece la consideración del estilo cognitivo. Muchos de los factores incluidos en los estudios convencionales de factores asociados son características que podrían, con justa razón, ser consideradas irrelevantes desde alguno o varios puntos de vista. Tal y como lo anotaba Tedesco (1992: 19), “(...) muchos sistemas de información resultan inoperantes no porque midan cosas superfluas en sí mismas sino porque son superfluas desde el punto de vista de las posibilidades de utilización de dicha información". No es éste el caso del estilo cognitivo, cuya consideración ofrece innegables ventajas para maximizar el efecto positivo de la acción educativa, particularmente en el orden pedagógico. Los estudios al respecto han mostrado una gran cantidad de elementos que favorecen el logro de aprendizaje de sujetos de uno u otro estilo. Así, el reconocimiento de las diferencias cognitivas en el salón de clase conduce necesariamente a una personalización de la acción pedagógica, que redunda inmediatamente en logros marcadamente mejores para la generalidad de los estudiantes.

\section{Referente teórico}

\section{El logro académico}

Entendemos por "logro académico" una categoría que intenta compendiar todo aquello que un estudiante alcanza como resultado directo de su exposición a un sistema educativo. Atendiendo a las funciones que normalmente se asignan a los sistemas educativos, los logros académicos son de muy diverso orden. Posibles dimensiones de logro son: la construcción de conocimientos, el desarrollo de habilidades, la formación de hábitos y actitudes, y la internalización de valores, entre otras (Moreno, 1998).

Como se desprende de su definición, el logro académico es una variable de naturaleza individual. Dentro de un sistema educativo dado, un individuo demuestra un cierto nivel de logro en cada una las dimensiones que el sistema defina como sus objetivos. El nivel se determina, por lo general, por medio de un juicio de valor o evaluación acerca de lo alcanzado por el estudiante, de acuerdo con criterios específicos de logros mínimos que deben ser alcanzados en cada momento de su trayectoria dentro del sistema.

Asumiremos, para lo que compete al presente estudio, que el logro académico está indicado, al menos en parte, por medio de dos tipos de evaluación. El primer tipo 
corresponde a una evaluación masiva de carácter estándar, realizada por instancias del sistema que no se encuentran involucradas directamente con el proceso pedagógico vivido por el estudiante. El segundo se trata de una evaluación personal y directa, realizada por los profesores, de cada estudiante, la cual hace parte del proceso pedagógico en el aula. Hablaremos brevemente de cada uno de ellos.

Con variaciones respecto de las denominaciones específicas de logro académico sobre las que centran su atención, los macroproyectos de evaluación masiva buscan obtener información general sobre la calidad de un determinado sistema educativo a partir de la construcción de pruebas estandarizadas de logro (logro cognitivo, logro de competencias básicas,...) y realizan aplicaciones muestrales o censales de las mismas. Este tipo de proyectos de evaluación tienen en nuestro país una historia bastante reciente. En el plano nacional, está el Sistema Nacional de Evaluación de la Calidad de la Educación (Proyecto Saber del Ministerio de Educación, desde 1992), con aplicaciones periódicas de pruebas de logro a grados que se alternan cada año. En el plano local, tenemos el proyecto de Evaluación de Competencias Básicas de la Secretaría de Educación del Distrito (SED), el cual, desde 1998, ha venido aplicando pruebas de competencias básicas en lenguaje, matemáticas, ciencias naturales $\mathrm{y}$, en algunos casos, comprensión y sensibilidad ciudadana, a la totalidad de los estudiantes de los grados $3^{\circ} 5^{\circ} 7^{\circ} \mathrm{y}, 9^{\circ}$ del sistema educativo de la capital del país. Será con información producida en este proyecto con la que trabajaremos esta dimensión del logro académico en el presente estudio.

Ahora bien, aunque una prueba objetiva y estandarizada proporciona información valiosa sobre lo aprendido por un estudiante en un momento dado de su trayectoria educativa, lo hace solamente con respecto a criterios generales del "deber ser" de un sistema educativo dado. Este hecho tiene sus ventajas y sus dificultades.

Dentro de las ventajas, la medida obtenida por este tipo de estudios es una medida generalizada, con pretensiones de objetividad. En este sentido, los grandes estudios de evaluación de la calidad de la educación hacen operacional la definición de ciertos logros mínimos, que en últimas se originarían en las demandas hechas por la sociedad al sistema educativo. Al ser una medida generalizada, hace para la determinación de estos logros mínimos una definición común para toda la población, lo que constituye una ventaja desde cierto punto de vista y, desde otro, una limitación, al ignorar las características particulares de los diferentes grupos sociales a los que se dirige.

La idea de la objetividad de las pruebas, además, reside, consideramos, en la intención de que el proceso de la resolución de los instrumentos esté desprovista de efectos "subjetivos", relativos a condiciones particulares, personales o circunstanciales del sujeto que los resuelve. Este punto, aunque resulta obviamente cuestionable desde lo que actualmente sabemos en los campos de la psicología y de la antropología cognitivas (en el sentido en que ello no es teóricamente posible) no deja por ello de ser deseable.

Algunas de las principales limitaciones de las medidas de logro académico obtenidas a través de la aplicación generalizada de pruebas objetivas ya han sido mencionadas. En primer lugar, debe reconocerse que los logros mínimos pueden no corresponder de manera exacta con los objetivos educativos planteados por un grupo social, una institución en particular o por un profesor específico. En consecuencia, si un estudiante resulta evaluado con niveles bajos de logro en este tipo de pruebas, esto no necesariamente significa que no haya aprendido, sino que posiblemente no ha aprendido lo que "debía", según un criterio externo a quienes agencian directamente su proceso de aprendizaje. En segundo lugar, está la crítica, en último termino irresoluble, acerca de la objetividad del proceso. En tercer lugar, está el problema de la precisión y la profundidad 
de la información obtenida: entre más medimos, en mayor cantidad de personas, menos sabemos acerca de cada cual.

Estos imponderables de las pruebas estandarizadas aplicadas para determinar la calidad de un sistema son obviados en su totalidad por el otro tipo de evaluación del logro que hemos considerado en este estudio: la evaluación del desempeño del estudiante por parte de sus profesores, la cual tiene como características básicas que: 1) toma en cuenta el logro de lo que, efectivamente, se propone como objetivo pedagógico y 2) si bien puede evaluar productos, toma necesariamente en cuenta el proceso seguido para alcanzar el logro. Como evaluación personalizada que es, su propósito no es indicar niveles institucionales o globales de calidad educativa, sino es la transformación del proceso a través de una acción pedagógica en el aula. De la misma forma, como evaluación pedagógica que es, proporciona información sobre la cual se puede actuar de manera directa, focalizada e inmediata. Las limitaciones de esta evaluación son las fortalezas de la otra: la imposibilidad de una medida generalizada para grandes grupos, y la influencia de factores subjetivos, relativos al maestro específico en los resultados de la evaluación.

Para la consecución de información relativa a este tipo de evaluación del logro, preguntamos a los profesores de matemáticas, lenguaje, ciencias naturales y ciencias sociales de los estudiantes de la muestra por el logro alcanzado por cada uno de estos en lo que iba del año lectivo que estaban cursando. Los resultados constituyen cuatro nuevos indicadores de logro académico que se yuxtaponen a los tres obtenidos de la aplicación de las pruebas de competencias.

En resumen, los indicadores de logro académico que el proyecto asumió para su análisis fueron: 1] los resultados de las Pruebas de Competencias Básicas (en matemáticas, lenguaje y ciencias naturales), aplicadas por la SED en octubre de 1999 a los grados $7^{0}$ y $9^{\circ}$ y 2] los juicios emitidos por los profesores de las áreas de matemáticas, lenguaje, ciencias naturales y ciencias sociales acerca del desempeño de los estudiantes durante el presente año lectivo. Esto significa, en términos reales, que el proyecto obtuvo información sobre siete indicadores del logro académico alcanzado por los estudiantes.

\section{El estilo cognitivo}

Definimos estilo cognitivo como el modo habitual o típico de una persona para resolver problemas, pensar, percibir y recordar (Tennant 1988). Existen muchos tipos diferentes de estilos cognitivos, técnicamente conocidos como «dimensiones». Casi todas estas dimensiones se nombran por medio de polaridades que reflejan los extremos a los que cada estilo cognitivo tendería. El presente proyecto de investigación se centra en una dimensión que tiene inmediata aplicación en la enseñanza y el aprendizaje: la dimensión de independencia del medio/sensibilidad al medio ${ }^{195}$. En su expresión cognitiva, la independencia/sensibilidad al medio consiste en la tendencia de algunas personas a fragmentar y asignarle una estructura propia a la información disponible para realizar una tarea o resolver un problema; en contraste con la tendencia de otras, a aproximarse a esta información de manera holística, manteniéndola en el mismo contexto en que ella está disponible. La primera de estas tendencias es la del independiente del medio y la segunda es la del sensible al medio. En suma, mientras el sujeto independiente del medio abstrae y descontextualiza, el sensible al medio concreta y contextualiza.

Desde otro punto de vista, esta dimensión de estilo cognitivo puede describirse en términos del tipo de respuesta social que el individuo privilegia para relacionarse e

\footnotetext{
${ }^{195}$ Desarrollada por Herman Witkin y su grupo de investigadores a finales de los años 1940 y principios de los 50, bajo el nombre de independencia/dependencia de campo (Witkin y Goodenough, 1981). 
interactuar con sus congéneres. Así, mientras los independientes del medio son sujetos que establecen una clara separación entre ellos y su entorno, incluidas las personas que los rodean, los sensibles al medio son personas que se perciben y definen a sí mismas como parte de un todo que los abarca. Esto hace que los sujetos independientes del medio sean individuos autónomos e independientes, con tendencia a manejar sus relaciones sociales sobre la base de objetivos impersonales más que sobre la base de aspectos como la adscripción al grupo o la empatía personal. En contraste, los sensibles al medio son personas que no establecen límites claros entre ellos y su entorno físico o social. Son individuos adscriptivos y sociables, con tendencia a manejar sus relaciones interpersonales sobre la base de niveles de empatía personal más que sobre la base de objetivos de trabajo ${ }^{196}$.

En general, puede considerarse que la tendencia hacia la independencia o hacia la sensibilidad es el resultado de la interacción de factores biológicos, de orden genético y endocrino, y de factores ambientales: físicos, sociales y culturales que constituyen el entorno en el que nacemos y crecemos. Mientras los primeros representan las condiciones iniciales del desarrollo, los segundos son los elementos a los que respondemos de manera adaptativa.

Así mismo, la independencia/sensibilidad al medio tiene una descripción neurofisiológica bastante precisa. Sabemos que cada polaridad del estilo puede describirse en términos del tipo de procesamiento que domina en el funcionamiento cerebral de cada individuo. Concretamente, se ha encontrado que mientras la independencia del medio supone altos niveles de lateralización hemisférica y un manejo de las funciones cerebrales muy especializado, la sensibilidad al medio está relacionada con menores niveles de lateralización hemisférica y un manejo más integral y compartido de las funciones cerebrales.

Muchas variables han sido vinculadas con la independencia/sensibilidad al medio. Entre ellas pueden mencionarse el sexo, la edad, la estructura de la familia y de la autoridad familiar, modalidades de crianza, y la pertenencia a grupos culturales específicos, por citar solo una pocas. El presente trabajo toma en consideración algunas, ya no solamente en su asociación con el estilo cognitivo, sino en su relación con el logro académico de los estudiantes. La idea que subyace a este tipo de análisis es que la explicación de la asociación entre diferentes factores individuales y familiares, y el logro académico se da por la mediación del estilo cognitivo entre los factores y el mismo logro.

\section{La hipótesis}

Nuestro modelo educativo, presente tanto en la educación oficial como en la privada, es, en esencia, un modelo graduado, grupal, jerarquizado, en el que se valora en grado sumo la abstracción, en el que predominan interacciones pedagógicas de tipo "frontal", y en el cual se pone énfasis en información básicamente visual y auditiva para el logro del aprendizaje. Frente a este modelo, nuestra hipótesis es la presencia de un sesgo en el sistema educativo que favorece el logro del sujeto independiente del medio y desfavorece al sensible.

Desde el punto de vista macroeducativo, la verificación de esta hipótesis explica los bajos logros académicos persistentemente encontrados en la población colombiana. En efecto, estudios previos han demostrado que los estudiantes colombianos muestran una tendencia persistente hacia el polo de la sensibilidad al medio, tendencia que resulta

\footnotetext{
${ }^{196}$ Para una descripción más detallada del funcionamiento cognitivo y socioafectivo de cada una de las polaridades de esta dimensión de estilo cognitivo, véase Hederich y Camargo (1993): Hederich y cols. (1995) y Hederich y Camargo (1999a).
} 
particularmente acentuada en grupos culturales fluviomineros (litorales y valles de los principales ríos) y, particularmente, en el litoral pacifico colombiano (Hederich y Camargo, 1995, 1999b). Así, una verificación de esta hipótesis aportaría una explicación positiva de los bajos niveles de calidad de la educación, por la vía de una inadecuación básica entre los modelos educativos y pedagógicos y las características cognitivas y culturales de la población.

En el origen de esta desigualdad está la presuposición psicopedagógica de que todos los estudiantes aprenden de la misma forma y, por tanto, requieren de los mismos insumos para su proceso de aprendizaje. Esta presuposición es falsa y su modificación es una condición necesaria, y además factible, para el mejoramiento de los niveles de calidad de la educación en Colombia.

\section{Metodología}

\section{Diseño}

La presente investigación se puede considerar como una observación, de tipo probabilístico y multivariado, en la que se obtiene información acerca de los niveles de logro académico (indicados, como hemos dicho, por los resultados en pruebas objetivas y evaluaciones pedagógicas de los maestros regulares), los estilos cognitivos en la dimensión de independencia/sensibilidad al medio, y una serie de variables potencialmente asociadas con cada uno de estos constructos.

En relación con el logro académico, éste se encuentra señalado por dos grupos de indicadores: 1] los puntajes obtenidos por los sujetos de la muestra en las pruebas de competencias básicas en matemáticas, lenguaje y ciencias naturales aplicadas por el proyecto de Evaluación de Competencias Básicas de la Secretaría de Educación del (1999) y 2] los niveles de rendimiento académico de los sujetos de la muestra, de acuerdo con la valoración que de éste hacen sus profesores de matemáticas, lenguaje, ciencias naturales y ciencias sociales, en una escala que va desde "deficiente", pasando por "aceptable" y "superior", hasta "excelente". Igualmente, el estilo cognitivo se encuentra indicado por los puntajes obtenidos en la Prueba de Figuras Enmascaradas (EFT), instrumento que mide la velocidad de reestructuración perceptual, principal indicador del nivel de independencia del medio. El instrumento alcanza niveles de confiabilidad de 0.91 (Hederich y Camargo, 1999).

Interviene también en el análisis un conjunto de variables asociables tanto al logro académico como al estilo cognitivo. Las tablas 1 y 2 presentan los factores potencialmente asociados con la relación estilo cognitivo/logro académico que fueron considerados en el presente estudio. Para la recolección de esta información se aplicó un cuestionario a la totalidad de los estudiantes participantes. 
Tabla 1. Variables vinculadas con el sujeto en cuanto individuo

\begin{tabular}{|c|c|c|c|}
\hline & & & INDICADOR \\
\hline \multirow{6}{*}{$\begin{array}{l}\text { Indicadores } \\
\text { individuales, } \\
\text { familiares } \\
\text { y culturales. }\end{array}$} & \multirow{2}{*}{$\begin{array}{l}\text { Perfil } \\
\text { organísmico }\end{array}$} & Género & Autorreporte \\
\hline & & 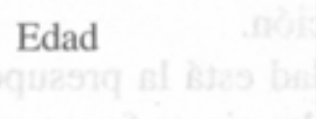 & $\begin{array}{l}\text { Indicada por la fecha } \\
\text { de nacimiento reportada }\end{array}$ \\
\hline & \multirow{4}{*}{$\begin{array}{l}\text { Influencias } \\
\text { sociales y } \\
\text { culturales }\end{array}$} & $\begin{array}{l}\text { Nivel } \\
\text { socioeconómico }\end{array}$ & $\begin{array}{l}\text { Influencias sociales y culturales } \\
\text { Indicado por la estratificación de } \\
\text { servicios más frecuente en la } \\
\text { manzana en la que se ubica la } \\
\text { residencia de la familia } \\
\text { del estudiante. }\end{array}$ \\
\hline & & $\begin{array}{l}\text { Nivel } \\
\text { sociocultural }\end{array}$ & $\begin{array}{l}\text { Indicado por los niveles } \\
\text { educativos del padre y la madre. }\end{array}$ \\
\hline & & Grupo familiar & $\begin{array}{l}\text { Estructura, extensión y tamaño } \\
\text { del grupo familiar. }\end{array}$ \\
\hline & & $\begin{array}{l}\text { Proveniencia } \\
\text { ecocultural } \\
\text { de la familia }\end{array}$ & $\begin{array}{l}\text { Lugar de nacimiento } \\
\text { del estudiante, de sus padres } \\
\text { y de sus abuelos }\end{array}$ \\
\hline
\end{tabular}




\section{Población y muestra}

La población de este estudio son los estudiantes de los colegios oficiales de la ciudad de Bogotá, en los grados $8^{\circ}$ y $10^{\circ}$ de la educación básica y media. La elección de estos grados obedeció a criterios eminentemente prácticos. En la medida en que el proyecto

Tabla 2. Variables vinculadas con el sujeto en cuanto estudiante

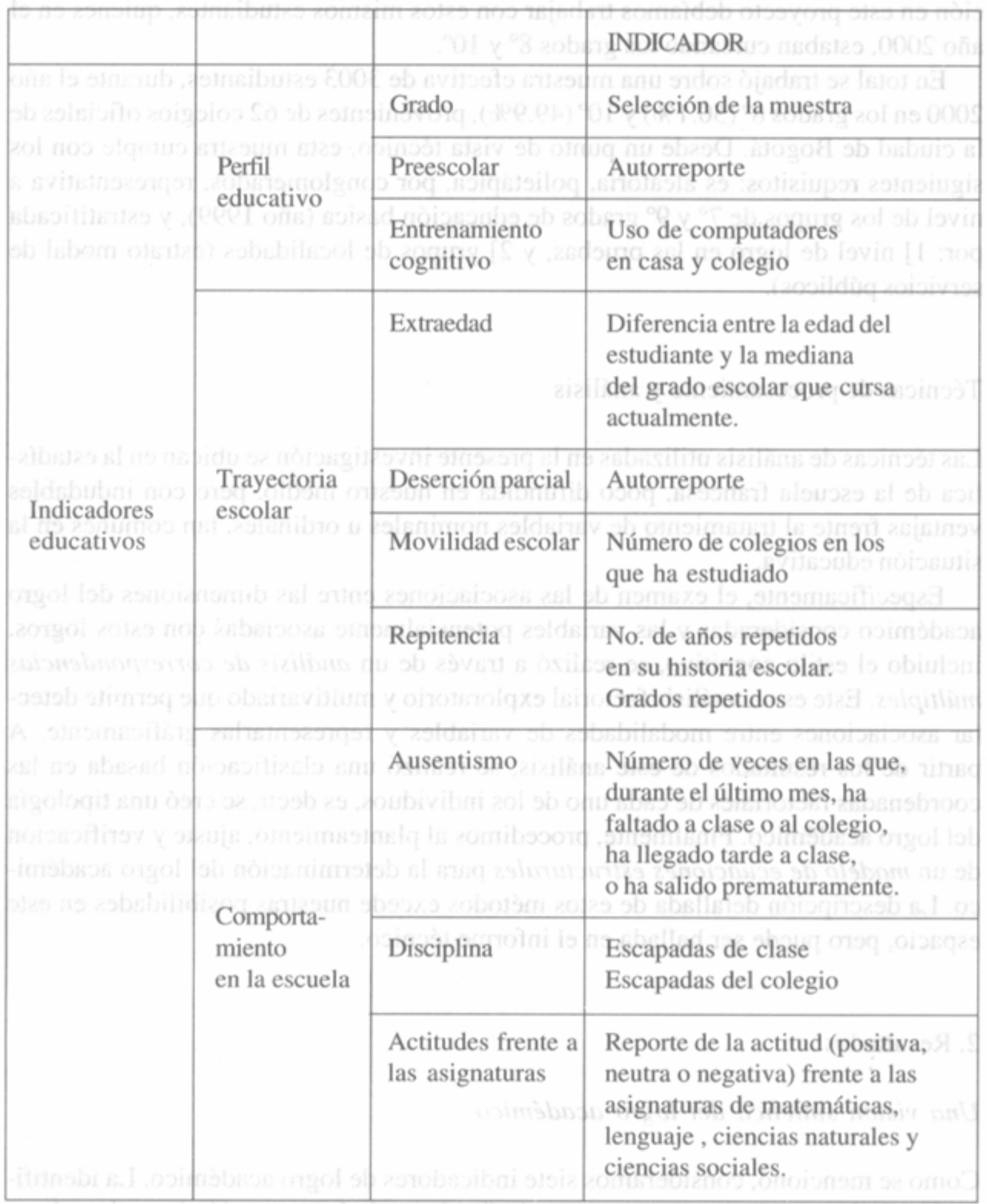


debía trabajar sobre la base de información de logro académico, asumimos como uno de sus indicadores los resultados del proyecto de Evaluación de Competencias Básicas en lenguaje, matemáticas y ciencias de la Secretaría de Educación del Distrito. Este fue un proyecto de evaluación censal realizado con los estudiantes que, en octubre de 1999, estaban cursando los grados $7^{\circ}$ y $9^{\circ}$. A fin de poder utilizar tal información en este proyecto debíamos trabajar con estos mismos estudiantes, quienes en el año 2000, estaban cursando los grados $8^{\circ}$ y $10^{\circ}$.

En total se trabajó sobre una muestra efectiva de 3003 estudiantes, durante el año 2000 en los grados $8^{\circ}(50.1 \%)$ y $10^{\circ}(49.9 \%)$, provenientes de 62 colegios oficiales de la ciudad de Bogotá. Desde un punto de vista técnico, esta muestra cumple con los siguientes requisitos: es aleatoria, polietápica, por conglomerados, representativa a nivel de los grupos de $7^{\circ}$ y $9^{\circ}$ grados de educación básica (año 1999), y estratificada por: 1] nivel de logro en las pruebas, y 2] grupos de localidades (estrato modal de servicios públicos).

\section{Técnicas de procesamiento y análisis}

Las técnicas de análisis utilizadas en la presente investigación se ubican en la estadística de la escuela francesa, poco difundida en nuestro medio, pero con indudables ventajas frente al tratamiento de variables nominales $u$ ordinales, tan comunes en la situación educativa.

Específicamente, el examen de las asociaciones entre las dimensiones del logro académico consideradas y las variables potencialmente asociadas con estos logros, incluido el estilo cognitivo, se realizó a través de un análisis de correspondencias múltiples. Este es un análisis factorial exploratorio y multivariado que permite detectar asociaciones entre modalidades de variables y representarlas gráficamente. A partir de los resultados de este análisis, se realizó una clasificación basada en las coordenadas factoriales de cada uno de los individuos, es decir, se creó una tipología del logro académico. Finalmente, procedimos al planteamiento, ajuste y verificación de un modelo de ecuaciones estructurales para la determinación del logro académico. La descripción detallada de estos métodos excede nuestras posibilidades en este espacio, pero puede ser hallada en el informe técnico.

\section{Resultados}

\section{Una visión sintética del logro académico}

Como se mencionó, consideramos siete indicadores de logro académico. La identificación de las asociaciones entre estos indicadores de logro académico asignados a cada estudiante, se llevó a cabo por medio de un análisis de correspondencias múltiples.

Los resultados de este análisis muestran dos dimensiones importantes y separables, representadas por los dos primeros factores del análisis, con valores propios de 0.2934 y 0.2090 respectivamente. La síntesis entre estos dos factores se presenta en el recuadro 1. 
Recuadro 1. Plano factorial del ogro académico

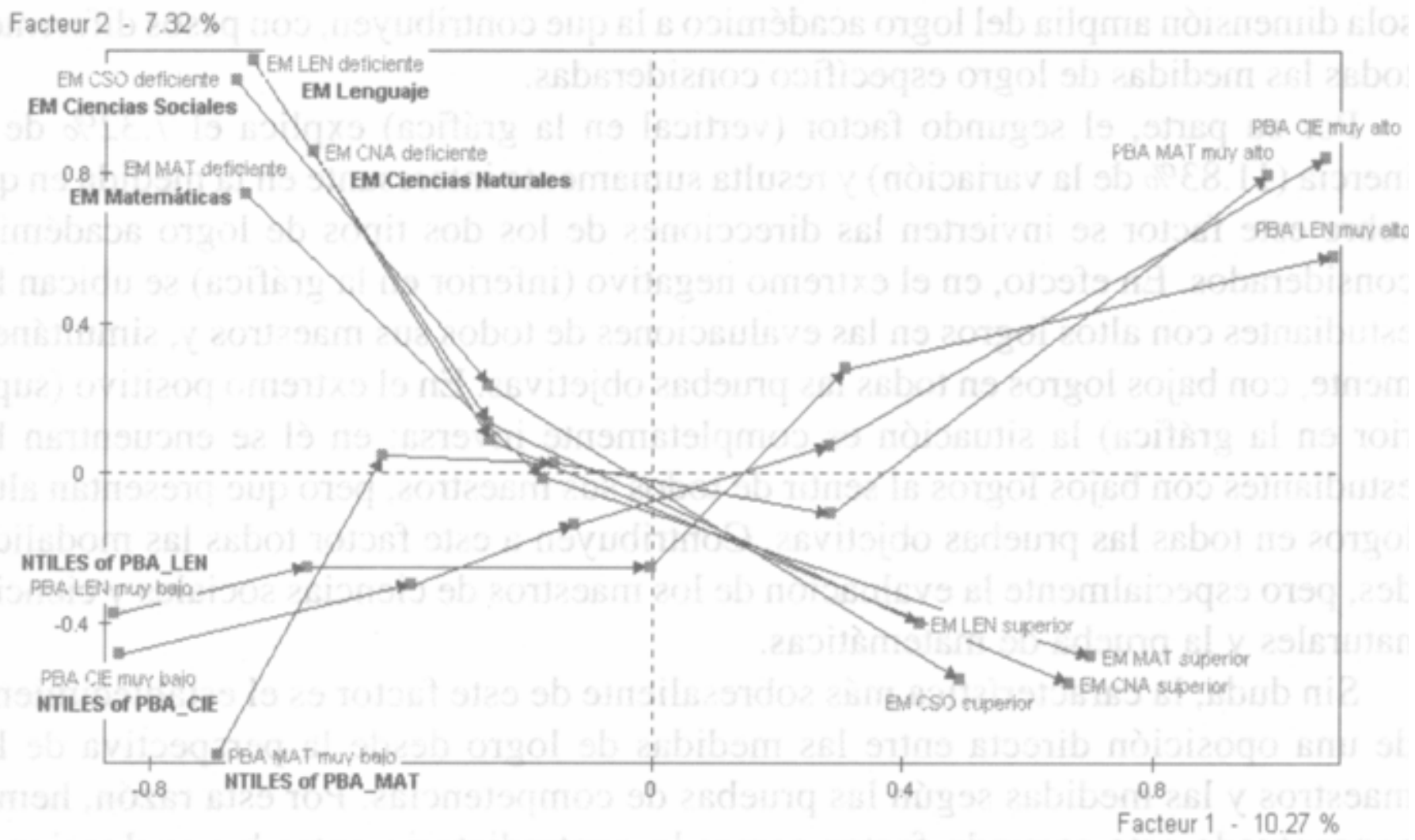

Los resultados del análisis muestran dos dimensiones importantes y separables representadas as por los dos primeros factores, con valores propios de 0.2934 y 0.2090 respectivamente. La gráfica presenta la síntesis de estos dos factores.

El primer factor (horizontal en la gráfica) explica el $10.27 \%$ de la inercia $(27.74 \%$ de la variación ${ }^{197}$ ) y polariza en extremos opuestos los resultados de todas las pruebas de competencias (matemáticas, lenguaje y ciencias naturales) y los resultados de todas las evaluaciones realizadas por los maestros (matemáticas, lenguaje, ciencias naturales y ciencias sociales) en el mismo sentido. Así, en el extremo izquierdo (negativo) se encuentran todos los bajos logros, tanto en las pruebas de competencias como en las evaluaciones de los maestros, y en el extremo derecho (positivo) se ubican todos los altos logros. Contribuyen de forma especialmente alta a este factor los resultados de las diferentes pruebas objetivas y, en menor medida, las evaluaciones de los maestros ${ }^{198}$.

Este es, pues, un factor en el que se asocian todas las medidas del logro académico entre sí, en una dirección consistente. Por esta característica, denominamos al primer factor como aquello en lo cual coinciden todas las medidas del logro: "lo común en todas las evaluaciones". Al sintetizar en una dirección consistente todos los indicadores, podríamos considerar que este factor representa, en su forma más general, una sola dimensión amplia del logro académico a la que contribuyen, con pesos diferentes, todas las medidas de logro específico consideradas.

\footnotetext{
${ }^{197}$ Nota estadística: Los porcentajes de variación, análogos al porcentaje de varianza explicada, se calculan sobre la base de los "valores propios" de cada factor.

${ }^{198}$ Por razones de espacio omitiremos en el presente artículo los valores de prueba, los niveles de significación y, en general, los indicadores estadísticos más especializados. Sugerimos al lector interesado remitirse al informe técnico.
} 
Por su parte, el segundo factor (vertical en la gráfica) explica el $7.32 \%$ de la inercia (11.83\% de la variación) y resulta sumamente interesante en la medida en que sobre este factor se invierten las direcciones de los dos tipos de logro académico considerados. En efecto, en el extremo negativo (inferior en la gráfica) se ubican los estudiantes con altos logros en las evaluaciones de todos sus maestros y, simultáneamente, con bajos logros en todas las pruebas objetivas. En el extremo positivo (superior en la gráfica) la situación es completamente inversa: en él se encuentran los estudiantes con bajos logros al sentir de todos sus maestros, pero que presentan altos logros en todas las pruebas objetivas. Contribuyen a este factor todas las modalidades, pero especialmente la evaluación de los maestros de ciencias sociales y ciencias naturales y la prueba de matemáticas.

Sin duda, la característica más sobresaliente de este factor es el establecimiento de una oposición directa entre las medidas de logro desde la perspectiva de los maestros y las medidas según las pruebas de competencias. Por esta razón, hemos conceptuado este segundo factor como: lo contradictorio entre las evaluaciones. ¿Qué contrapone tan frontalmente los dos tipos de evaluación? La respuesta deberá, en atención al rigor, esperar algunas páginas; baste decir por ahora que son ciertas características de comportamiento social, indisolublemente asociadas con el estilo cognitivo, las que marcarán las diferencias que aquí observamos.

Vistos los resultados en conjunto, tal y como se observa del análisis de las trayectorias de los resultados de cada evaluación, existe una evidente similitud entre las trayectorias de las pruebas de competencias, por un lado, y de las evaluaciones de todos los maestros, por el otro: las trayectorias de las pruebas de competencias, en todas las áreas (matemáticas, lenguaje y ciencias naturales) son evidentemente similares, partiendo desde el extremo inferior izquierdo del plano factorial, que representa los más bajos logros, y llegando al extremo superior derecho, que representa los más altos logros. Lo mismo sucede con las trayectorias de todas las evaluaciones realizadas por los maestros de las diferentes áreas, las cuales parten del extremo superior izquierdo para desembocar en el extremo inferior derecho. Esto revela una estructura de asociaciones que privilegia el tipo de evaluación (o si se quiere, la fuente de la información), por encima de las particularidades de dominio específico.

En relación con las pruebas de competencias, estos resultados señalan una evidente asociación entre las tres pruebas objetivas de matemáticas, lenguaje y ciencias, que permite suponer la presencia de un factor común subyacente a las tres, el cual explicaría la similitud. Esto es, en alguna medida, las tres pruebas evalúan lo mismo. Esta fuerte asociación puede tener su explicación en la forma como el proyecto de Evaluación de Competencias Básicas (ECB) concibió estas competencias. Tal y como se explica:

"En la experiencia escolar estas competencias [matemáticas, lenguaje y ciencias] aparecen entrelazadas; así por ejemplo, los problemas en matemáticas se plantean y comprenden en enunciados Lingüísticos; y las ciencias, como la física y la química, utilizan procedimientos matemáticos. Posiblemente, no existe una tarea pura de Lenguaje, de matemática o de ciencias, a pesar de que cada una de estas áreas exige competencias particulares. Surge entonces la pregunta por las relaciones entre las competencias y una supuesta independencia, así como la hipótesis de que existen operaciones cognitivas comunes a todas ellas. La literatura sobre el tema plantea varios puntos de vista al respecto (SED, 1999:15-16)".

Es claro, a partir del formato que se asumió para configurar la prueba y, también, a partir de nuestros resultados, que la alternativa por la que se optó en el proyecto de ECB fue el privilegiar las relaciones entre las competencias de las diferentes áreas, con el menoscabo de la especificidad de dominio de cada una de ellas. La opción produjo, a nuestro juicio, una cierta homogenización de los desempeños por evaluar, y los sintetizó, 
posiblemente, en una competencia comunicativa general o específica (encontrar información relevante en un periódico, por ejemplo). Los mejores, aunque aún discretos, desempeños en el área de lenguaje apoyan esta hipótesis.

Ahora, en relación con la evidente similitud entre las evaluaciones de los cuatro maestros de las áreas consideradas, los resultados indican que, al igual que en el caso de las pruebas, por encima de las particularidades de cada asignatura, los cuatro maestros evalúan, cada estudiante de la misma forma: siempre "superior" "aceptable" o "deficiente" para todas las áreas. Desaparecen de nuevo aquí las particularidades de las áreas consideradas y las posibilidades de que un estudiante dado sobresalga en un contenido específico y tenga un desempeño aceptable o deficiente en otros. ¿Cuál puede ser el factor común a las cuatro evaluaciones de los maestros? La respuesta deberá esperar aún unas páginas, pero la conjetura evidente es que, junto con los logros o las competencias específicas del estudiante en un área, lo que los maestros evalúan son ciertos rasgos de comportamiento escolar independientes del área específica considerada.

\section{Las variables que caracterizan el logro académico}

Caracterización global de los ejes factoriales por las variables ilustrativas

El examen de las asociaciones entre el logro académico y los factores potencialmente asociados con este logro se realizó a partir de su inclusión como variables ilustrativas en el plano factorial del logro académico, que resultó del análisis de correspondencias múltiples cuyos resultados ya se discutieron.

Una visión general de la ubicación de algunas de las modalidades más alejadas del centro, esto es, con mayor poder de discriminación del logro, se presenta en el recuadro 2.

Recuadro 2. Ubicación de algunas modalidades ilustrativas en plano factorial del logro académico

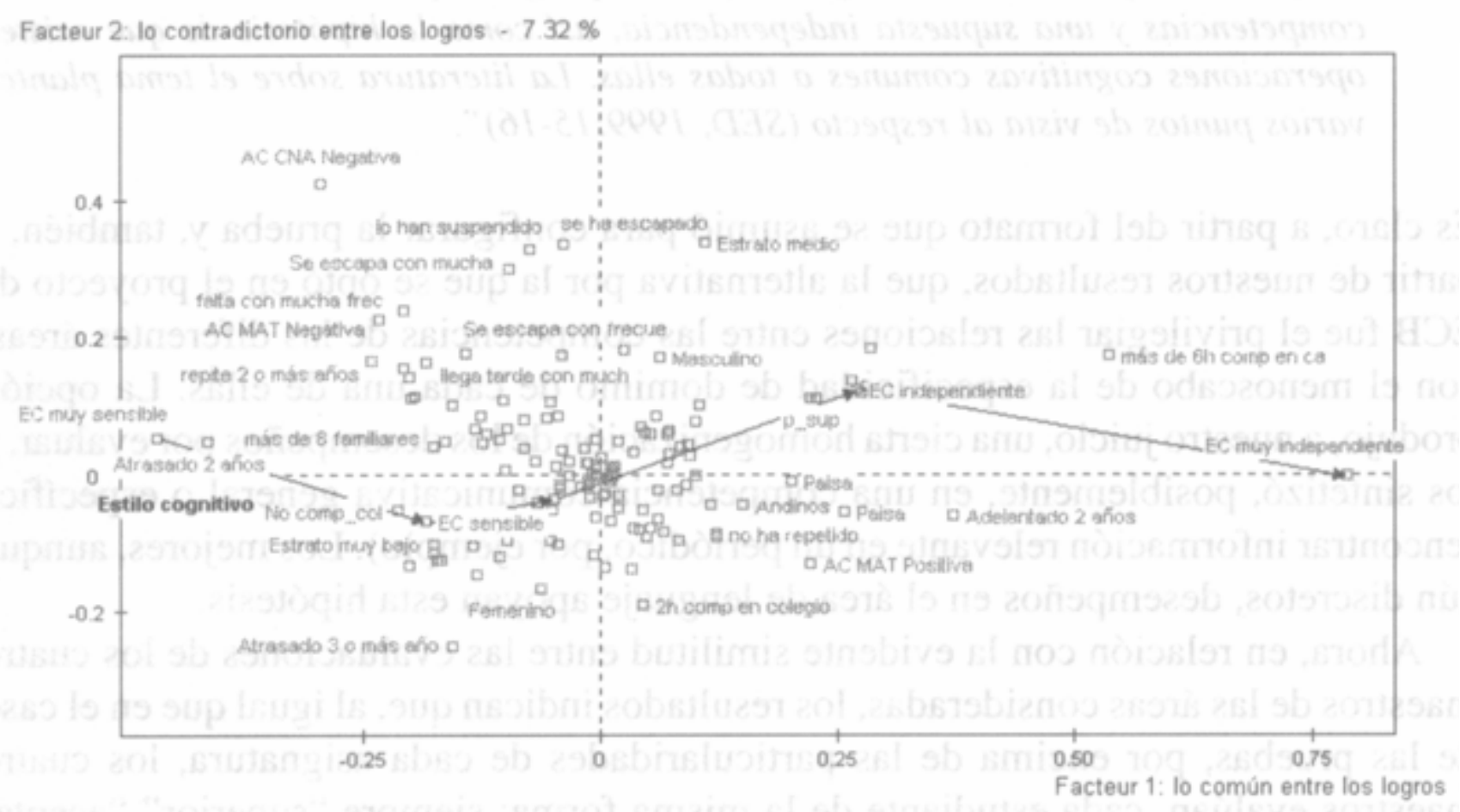


La gráfica ilustra la ubicación de algunas de las modalidades ilustrativas en el plano factorial del logro académico. Para su interpretación, recuerdese que los sitios en los que se ubican los valores representan su nivel de aproximación con las direcciones generales marcadas en el plano factorial de los logros académicos. Esto es, las modalidades ubicadas en el extremo superior derecho, por ejemplo, están asociadas con altos logros en las pruebas de competencia (tendencia derecha y tendencia superior) y en cierta contradicción con las evaluaciones de los maestros (tendencia superior).

El examen de las variables que caracterizan el primer factor (horizontal en el plano muestra una gran cantidad de modalidades ilustrativas que presentan asociaciones significativas con el factor. En términos generales, y en orden relativo de importancia, el extremo izquierdo del primer factor, que representa los bajos logros en todos los indicadores queda caracterizado por: 1] un estilo cognitivo muy sensible o sensible, 2] actitudes neutras o negativas frente a las matemáticas, 3] una alta repitencia (2 o más años), 4] una alta extraedad (2 o más años), 5] ausencia de uso de computadores, 6] comportamientos ausentistas (faltan a clase y llegan tarde con mucha frecuencia), 7] niveles socioculturales bajos (padre y madre con primaria incompleta), 8] estratos socioeconómicos bajos o muy bajos, 9] género femenino, y 10] familia amplia y extensa (más de 8 familiares). Otras variables que muestran asociación significativa con los bajos logros, aunque menos fuerte, son 11] haber estudiado sólo en el colegio actual y 12] comportamientos relativamente "disciplinados": (escaparse de clase sólo muy rara vez).

En el extremo opuesto, es decir, en el sentido positivo del factor 1, caracterizado por altos logros en todas las evaluaciones, se ubican, también en orden de significación, 1] estilo cognitivo muy independiente o independiente, 2] actitudes positivas frente a las matemáticas y las ciencias naturales, 3] ausencia de repitencia, 4] alta frecuencia de uso de computadores, especialmente en casa, 5] niveles socioculturales relativamente altos (padres con educación superior) y 6] niveles socioeconómicos relativamente altos (estrato medio-bajo). Se asocian también a esta dirección: 7] comportammentos escolares no ausentistas, 8] cierta proveniencia regional andina-santandereana o paisa ${ }^{199}$, 9] subedad (por oposición a extraedad, es decir, estar. adelantado dos años), 10] el género masculino, y 11] la convivencia en grupos familiares pequeños.

Una visión sintética de los dos sentidos muestra que el estilo cognitivo es la variable que en mayor medida discrimina diferencias en el primer factor del logro académico, sobre el cual coinciden todas las evaluaciones: los individuos de estilo independiente obtienen mejores logros. Al estilo cognitivo le siguen actitudes positivas hacia las matemáticas y las ciencias naturales, la trayectoria escolar (poca repitencia, y subedad), alto entrenamiento cognitivo (uso de computadores), escaso ausentismo $y$, finalmente el género del estudiante y algunos aspectos familiares y culturales del mismo.

En relación con el segundo factor, como se recordará, éste tiene el interés especial de que invierte los dos tipos de evaluación que hemos considerado: los resultados de las pruebas de competencias básicas y las evaluaciones de los diferentes maestros, toman sobre este factor direcciones contrapuestas.

En el extremo inferior, caracterizado por niveles altos en las evaluaciones de sus maestros pero bajos en las pruebas de competencia, se discriminan estudiantes 1] de género femenino, 3] sin repitencia, 4] cumplidores de la disciplina escolar (no se escapan

\footnotetext{
${ }^{199}$ Aludimos aquí a regiones colombianas desde la perspectiva de la Regionalización Ecocultural del país, que distingue 9 regiones: andina central, andina meridional, santandereana, litoral caribe, litoral pacifico, paisa, fluviominera del Valle del Cauca, fluviominera del Magdalena alto y fluviominera del Magdalena medio. Para una explicación más detallada véase nuestro trabajo publicado en 1999(b).
} 
de clase ni del colegio), 5] sin ausentismo, 6] con actitudes positivas hacia las matemáticas, el lenguaje y las ciencias naturales y 7] sin asistencia al preescolar. Con niveles de significación menores aparecen aspectos culturales tales como: 8] niveles socioculturales medios, 9] familias de tamaño intermedio (4 a 5 personas) y 10] proveniencia andina- santandereana.

\section{Recuadro 3. Pruebas de Competencias por Grupos de Estilo}

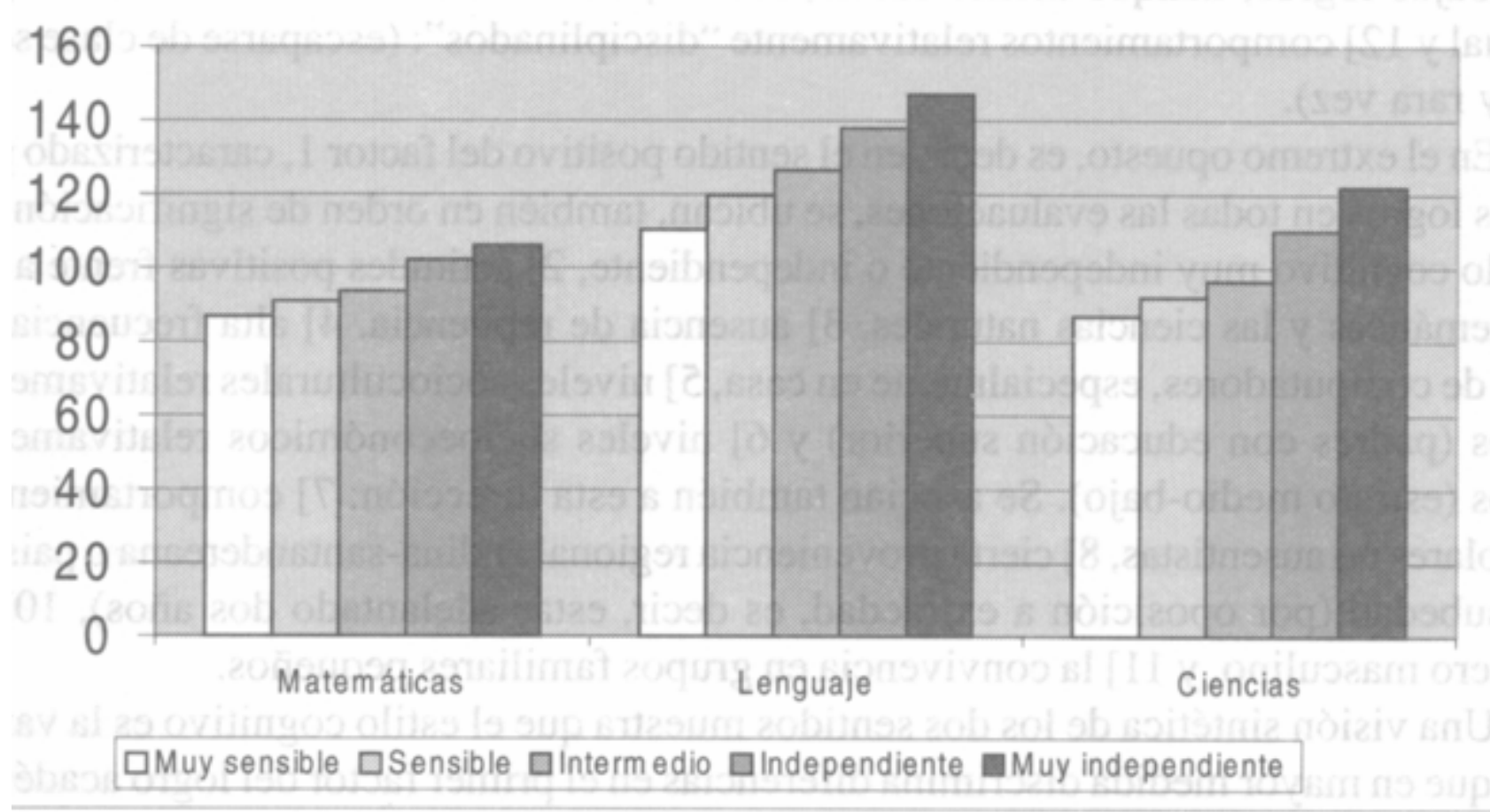

La gráfica muestra con claridad cómo los más altos puntajes en todas las pruebas de competencias corresponden al grupo de estilo cognitivo muy independiente. Los puntajes van decreciendo progresivamente hasta llegar al grupo de estudiantes muy sensibles, quienes presentan los puntajes más bajos en las tres áreas.

En el otro extremo, es decir, en el extremo superior del plano factorial, caracterizado por altos logros en las pruebas de competencia pero bajos logros según el juicio de los maestros, se encuentran estudiantes 1] varones, 2] con repitencia escolar, 3] en cierta oposición a la autoridad escolar ("indisciplina"), 4] ciertos niveles de ausentismo, 5] niveles socioculturales medio-altos, 6] asistencia al preescolar, 7] actitudes negativas hacia todas las asignaturas, 8] estilo cognitivo independiente, 9] alta movilidad escolar, y 10] estratos socioeconómicos medios.

Una visión general del segundo factor permite identificar al género como la variable que mejor se polariza entre los dos extremos de la dirección vertical del plano factorial. Así, mientras las mujeres obtienen bajos logros en las pruebas objetivas y altos en las evaluaciones de los maestros, a los varones les ocurre lo contrario: altos logros en competencias y bajos frente a sus maestros. Le siguen al género las variables de trayectoria escolar (repitencia) y comportamiento en la institución escolar (disciplina, actitudes y ausentismo) en una particular dirección: mayor repitencia, más problemas disciplinarios, actitudes más negativas hacia las asignaturas y mayor ausentismo se asocian con altos logros en las pruebas de competencias y con más bajas evaluaciones del maestros. 
Recuadro 4. Evaluaciones de Rendimiento por Grupos de Estilo

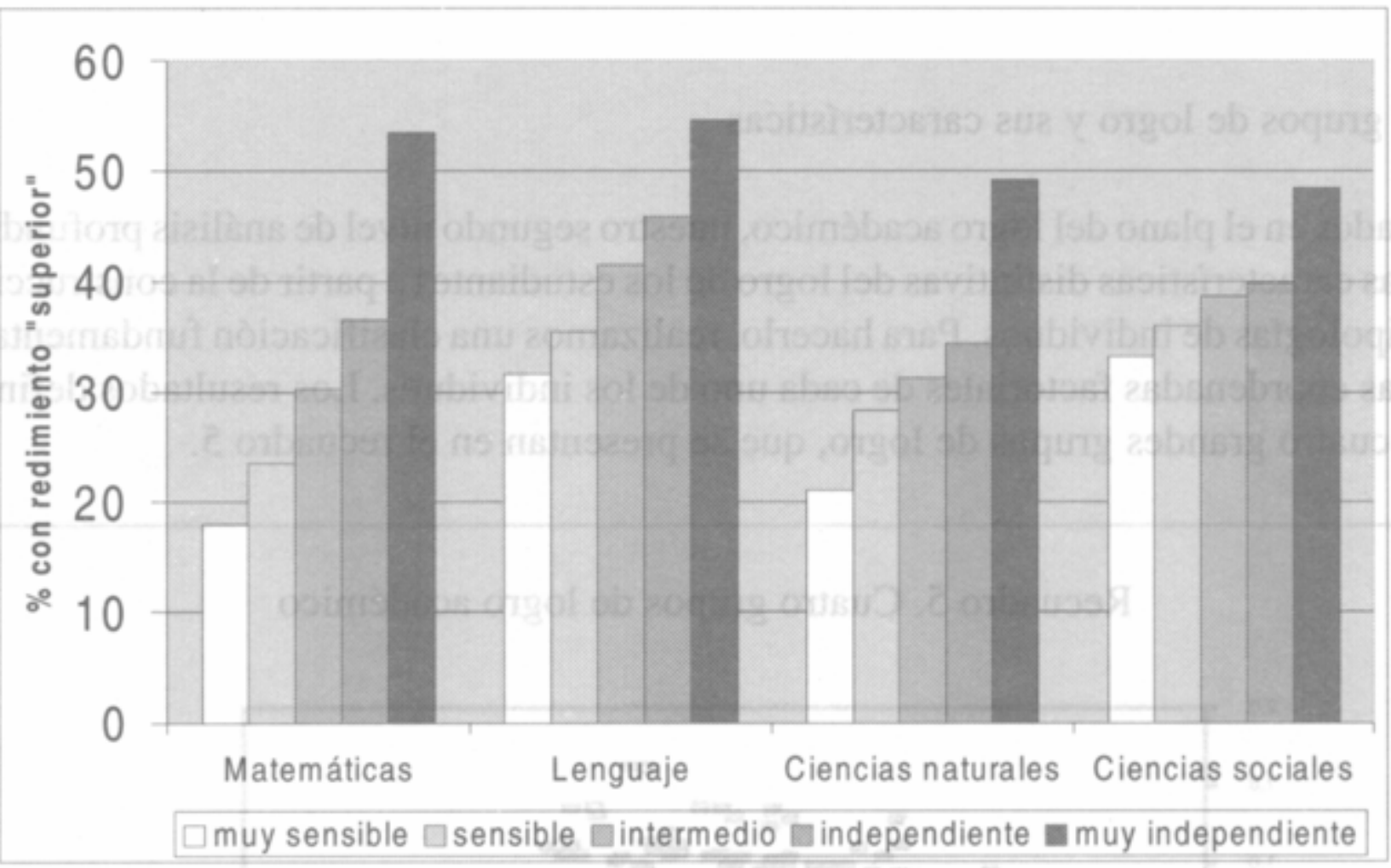

La gráfica muestra los porcentajes de estudiantes de cada uno de los grupos de estilo que poseen rendimientos superiores de acuerdo con sus maestros. Como se observa, es notoria la presencia de estudiantes muy independientes dentro de esta categoría de rendimiento en todas las áreas. El porcentaje va bajando hasta llegar al grupo de estudiantes muy sensibles, entre quienes se observan porcentajes mucho menores comparados con los rendimientos superiores. Nótese que la diferencia entre los grupos de estilo es especialmente contrastante en el área de matemáticas y bastante más discreta en el área de ciencias sociales.

Lo observado no deja dudas acerca de una estrecha relación entre el estilo cognitivo y el logro académico: entre mayor sea la independencia de campo del estudiante, mayores sus probabilidades de obtener altos logros, tanto en pruebas estandarizadas como en evaluaciones personales de los profesores.

Nuestros resultados se suman así a la lista ya extensa de hallazgos que evidencian esta asociación. En efecto, estudios realizados en países como Estados Unidos (Burwell, 1991; Kush, 1996), Reino Unido (Satterly, 1976), Francia (Barbe yTourrette, 1994), Canadá (Bialystock, 1992), Australia (Chandran, Treagust y Tobin, 1987), Jamaica (Leo Rhynie, 1985), China, Indonesia y Arabia Saudita (Abraham, 1985), Jordania (Al Nesir, Keenan y Langer, 1991) Turquía (Alptekin y Atakan, 1990) y España (Tinarejo y Páramo, 1997) han encontrado situaciones similares a la nuestra. En todos ellos se han entendido los resultados como evidencia de una situación de inequidad de los sistemas educativos en el sentido en que, al asumir como válida la premisa de que todos los individuos aprenden de la misma forma, están favoreciendo el logro de solo un grupo de estudiantes, al tiempo que están colocando una buena proporción de sus usuarios en situación de desventaja, con respecto de lo que pueden aprender. 


\section{Los grupos de logro y sus características}

Basados en el plano del logro académico, nuestro segundo nivel de análisis profundizó en las características distintivas del logro de los estudiantes a partir de la construcción de tipologías de individuos. Para hacerlo, realizamos una clasificación fundamentada en las coordenadas factoriales de cada uno de los individuos. Los resultados definieron cuatro grandes grupos de logro, que se presentan en el recuadro 5.

Recuadro 5. Cuatro grupos de logro académico

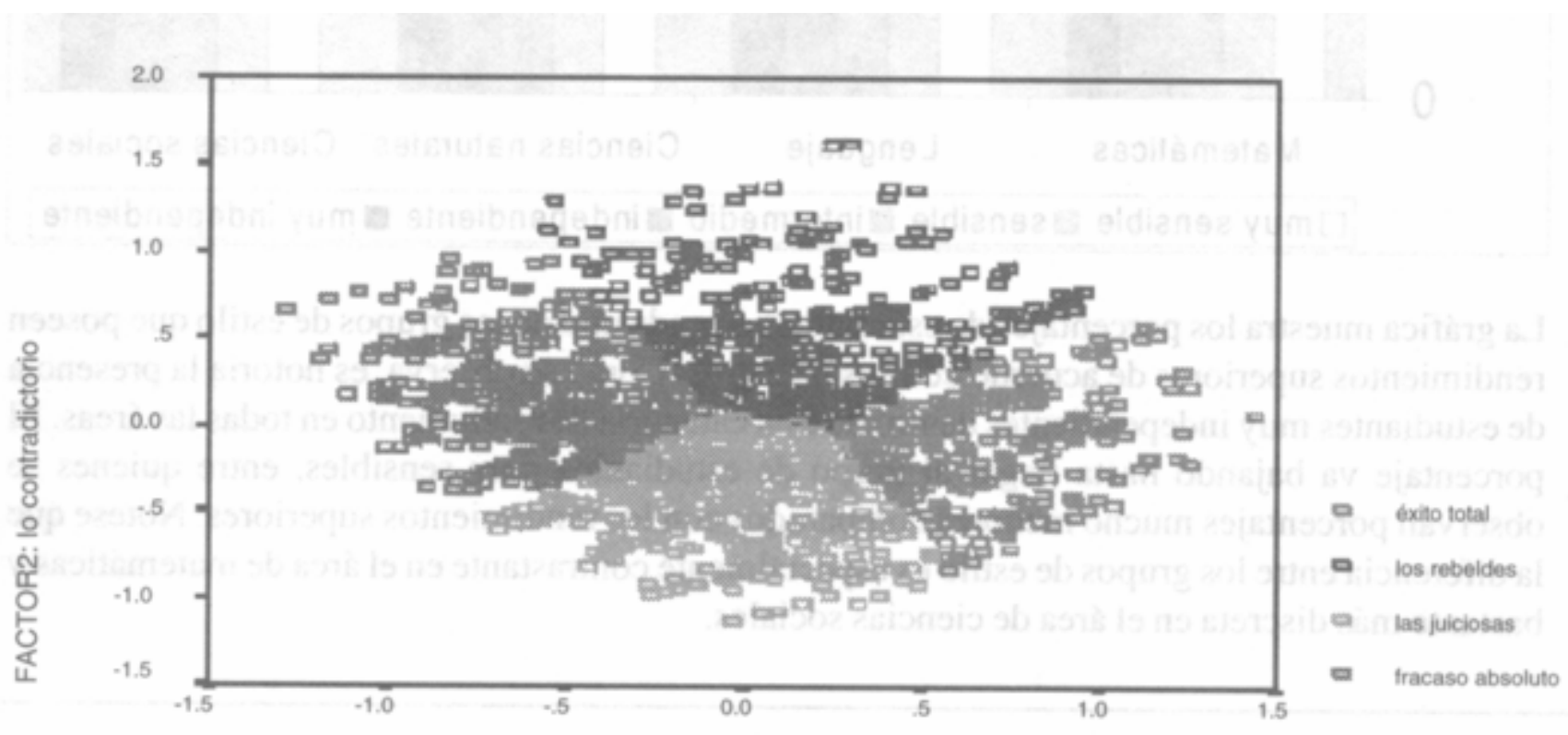

FACTOR1: lo común

La definición de los cuatro grupos de logro polariza y combina los dos ejes factoriales en todas sus posibilidades.

Al primer grupo, de bajas competencias y bajos rendimientos, pertenecen 531 sujetos que representan casi la tercera parte de la muestra (28,63\%). En razón a sus muy bajos logros lo hemos denominado como el grupo del "fracaso total".

Las características que en mayor medida identificaron a los estudiantes del primer grupo fueron, en orden relativo de significación, un estilo cognitivo muy sensible al medio, alta repitencia escolar, ausencia de uso de computadores en la casa y en el colegio, una extraedad de dos años o más, comportamientos escolares ausentistas, actitudes negativas hacia diferentes materias (especialmente, hacia las ciencias naturales y las matemáticas), corresidencia en familias extensas y de gran tamaño, proveniencia regional desconocida o fluviominera, estratos socioeconómicos muy bajos, y cierta preferencia por asignaturas de ciencias sociales y lenguaje.

Así mismo, en el segundo grupo, de bajas competencias en las pruebas pero altos rendimientos según los maestros, quedaron clasificados 553 estudiantes $(29.81 \%$,) por lo tanto, es similar en tamaño al anterior. Aparte de las peculiaridades de su logro académico, las características que en mejor medida identificaron a sus estudiantes fueron: un estilo cognitivo sensible al medio o intermedio, su género (en su mayoría femenino), bajos niveles de ausentismo, altos niveles de "disciplina escolar" (no se escapan de clase 
ni del colegio y no han sido suspendidos del mismo), y muy bajos niveles de movilidad escolar. De acuerdo con esas características lo hemos denominado como "los juiciosos" o mejor, incluyendo el género, el grupo de "las juiciosas".

El tercer grupo mostró altas competencias en las pruebas, pero bajos rendimientos (a juicio de los maestros). En tamaño, es minoritario frente a los dos anteriores (396 estudiantes: $21.35 \%$ ). Sus características distintivas fueron un estilo cognitivo independiente o muy independiente del medio, su género (en su mayoría masculino), ciertos niveles incipientes de ausentismo, una fuerte tendencia a tener problemas disciplinarios (a una buena proporción los han suspendido del colegio), y niveles relativamente altos de movilidad escolar. Este grupo es, en todas sus características, el opuesto al grupo de las "juiciosas", por lo que lo hemos denominado como "los rebeldes".

Finalmente, el cuarto grupo muestra altos logros en todas las evaluaciones, razón por la cual lo hemos denominado como el grupo del "éxito total". Está conformado por 375 estudiantes (20.22\%); y es exactamente opuesto al primero en la medida en que muestra altos logros en todos los indicadores de evaluación, sean estos asignados por los maestros o por las pruebas objetivas. Sus características distintivas fueron un estilo cognitivo muy independiente del medio, actitudes positivas, especialmente frente a la asignatura de matemáticas, ausencia de repitencia escolar, una situación de adelanto con respecto a la edad promedio del grupo escolar (subedad escolar), alta frecuencia de uso de computadores en casa y colegio; y niveles educativos altos en el padre y la madre.

Tabla 3. Los grupos de logro

\begin{tabular}{|l|l|l|l|}
\hline Grupo de logro & Logro en pruebas & $\begin{array}{l}\text { Logro según los } \\
\text { maestros }\end{array}$ & Estilo cognitivo \\
\hline Fracaso & Bajo & Bajo & Muy sensible \\
\hline Juiciosos & Bajo & Alto & Sensible-intermedio \\
\hline Rebeldes & Alto & Bajo & Independiente \\
\hline Éxito total & Alto & Alto & Muy independiente \\
\hline
\end{tabular}

Tal y como se observa, el examen de los estilos cognitivos característicos de cada uno de los cuatro grupos de logro muestra que el estilo difería entre ellos. En general, los grupos que mostraban mejor logro en las pruebas (éxito y rebeldes) tendían hacia la independencia, mientras que los grupos de bajo logro en pruebas tendían hacia la sensibilidad.

\section{Un modelo causal}

Como su nombre lo indica, los estudios de variables asociadas con el logro tienen como objetivo explorar las asociaciones entre un conjunto de variables y el logro académico. La idea subyacente a la elaboración de estos estudios, en términos de la gestión estratégica, es que, en alguna medida, algunos de estos factores asociados determinan el logro académico, por lo que al afectar los niveles de estos factores afectamos indirectamente el logro. Esta idea, sin embargo, no tiene base empírica para ninguno de los estudios de factores asociados con el logro, elaborados en el país. Esta es, tal vez, la razón principal por la cual el Departamento Nacional de Planeación califica los estudios de factores asociados realizados en el país como "incipientes" (DNP, 1999).

En efecto, una cosa es verificar asociaciones entre dos variables, otra es verificarlas entre múltiples variables, y otra, cualitativamente diferente de las dos anteriores, es verificar relaciones de causalidad entre variables. Cuando verificamos asociaciones, 
simplemente constatamos que dos fenómenos ocurren de manera simultánea, la mayoría de las veces; no más que esto. Por esta razón, cuando dos variables aparecen asociadas, no podemos saber cuál es la dirección de la asociación o, aún más, si efectivamente hay una dirección en esta asociación; pudiera ser, por ejemplo, que las dos variables no tuvieran una relación directa entre sí, sino que ambas resultaran ser consecuencia de una tercera variable oculta, que las explicara a las dos.

Para abordar este nivel de descripción, es necesario formular un modelo causal que establezca direcciones específicas en la asociación entre las variables y el logro. Tratamos ahora de predecir el logro académico en términos de variables que lo explican. La formulación de este tipo de modelos no ha sido intentada, hasta donde sabemos, para la predicción del logro académico en Colombia.

En el presente trabajo intentamos la construcción de un modelo causal, del estilo de "análisis de caminos" (path analysis). El método seguido para la construcción del modelo causal es el análisis de ecuaciones estructurales y su resultado se presenta en el recuadro 6. Este modelo, sin embargo no puede ser considerado terminado, en la medida en que sólo alcanza a vincular algunas de las variables que consideramos. El modelo logrado hasta el momento, sin embargo, muestra las características técnicas esperadas de convergencia, confiabilidad y estabilidad (ver más adelante los indicadores de prueba del modelo).

Recuadro 6. Modelo para la predicción del logro académico

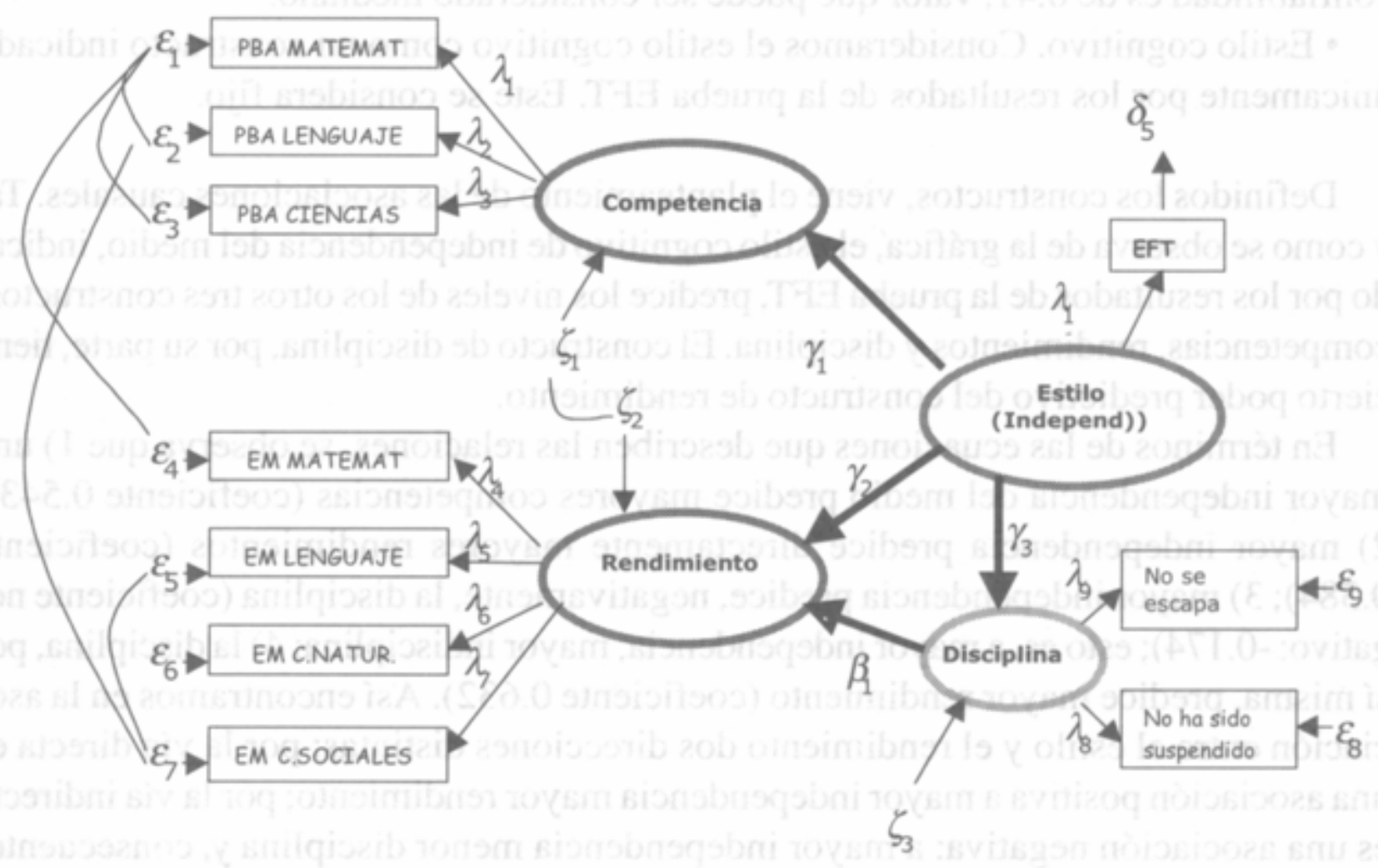

Las ecuaciones son:

La competencia depende únicamente del estilo:

Competencia 0.543 (EFT)

El rendimiento depende del estilo y de la disciplina:

Rendimiento $=0.384$ (EFT) +0.632 (Disciplina) 
La disciplina depende del estilo:

Disciplina $=-0.174$ (EFT)

Combinando (2) y (3):

Rendimiento $=0.274(\mathrm{EFT})$

El primer paso para la definición del modelo es el planteamiento de los constructos que consideraremos. Los constructos son:

- Competencia. Los resultados de las pruebas de competencias en matemáticas, lenguaje y ciencias naturales forman un constructo común, que llamamos "competencia". Su presencia fue clara, desde el análisis de correspondencias múltiples, realizado en el capítulo X. Su confiabilidad la cual es de 0.58 , que puede ser considerada buena (en el recuadro 21 se presentan los indicadores de los constructos).

- Rendimiento. Las evaluaciones de los maestros en todas las áreas forman un constructo común que denominamos "rendimiento". También en este caso era clara su presencia en las evaluaciones de los maestros. De nuevo su confiabilidad puede ser considerada buena (0.63).

- Disciplina. No haber sido suspendido del colegio y no escaparse del mismo pueden ser agrupados en un constructo común que llamamos "disciplina". Este tal vez es el más débil del modelo, en tanto solo está definido por dos variables "dummy". Su confiabilidad es de 0.41 , valor que puede ser considerado mediano.

- Estilo cognitivo. Consideramos el estilo cognitivo como un constructo indicado únicamente por los resultados de la prueba EFT. Este se considera fijo.

Definidos los constructos, viene el planteamiento de las asociaciones causales. Tal y como se observa de la gráfica, el estilo cognitivo de independencia del medio, indicado por los resultados de la prueba EFT, predice los niveles de los otros tres constructos: competencias, rendimientos y disciplina. El constructo de disciplina, por su parte, tiene cierto poder predictivo del constructo de rendimiento.

En términos de las ecuaciones que describen las relaciones, se observa que 1) una mayor independencia del medio predice mayores competencias (coeficiente 0.543); 2) mayor independencia predice directamente mayores rendimientos (coeficiente 0.384 ); 3 ) mayor independencia predice, negativamente, la disciplina (coeficiente negativo: -0.174 ); esto es, a mayor independencia, mayor indisciplina; 4) la disciplina, por sí misma, predice mayor rendimiento (coeficiente 0.632). Así encontramos en la asociación entre el estilo y el rendimiento dos direcciones distintas: por la vía directa es una asociación positiva a mayor independencia mayor rendimiento; por la vía indirecta es una asociación negativa: a mayor independencia menor disciplina y, consecuentemente, menor rendimiento.

\section{Análisis, conclusiones y recomendaciones}

Los resultados indican relaciones complejas entre los diferentes niveles de logro académico, el estilo cognitivo y las variables asociadas a esta relación. Por el primer factor, los estudiantes independientes, especialmente si lo son en grado extremo, muestran mejores competencias, mejores rendimientos, menor repitencia, menor extraedad, y actitudes más positivas hacia las matemáticas y las ciencias naturales; en el extremo opuesto del mismo factor, los estudiantes muy sensibles muestran menores competencias, menores rendimientos y mayores niveles de repitencia y extraedad. Esto confirma la presencia de un sesgo claro del sistema educativo en el sentido en que favorece el logro de los sujetos de uno solo de los estilos cognitivos: el de la 
independencia del medio. Se mantiene entonces nuestra primera hipótesis: el sistema educativo oficial favorece el logro de los estudiantes independientes, de dos formas relacionadas: 1] propiciando mejores aprendizajes en ellos, y 2] filtrando, manteniendo y, finalmente, excluyendo, en los grados inferiores, a los estudiantes de estilo cognitivo sensible.

Los resultados no se agotan aquí. Un examen del segundo factor muestra que algunas de las variables que aparecen caracterizándolo, también aparecen caracterizando el primer factor. ¿Cómo conciliar estos dos resultados aparentemente contradictorios? La respuesta debe buscarse para cada variable. Consideremos, por ejemplo, el género. Con el primer factor los varones tienen más altas competencias y rendimientos; con el segundo las mujeres, tienen más altos rendimientos y más bajas competencias; en conclusión: los varones tienen más altas competencias, pero la evaluación de los maestros resulta favorablemente sesgada para las muchachas: a pesar de que ellas muestren menores competencias, son mejor evaluadas por los profesores.

La variable de repitencia muestra un comportamiento similar. De acuerdo con el primer factor, esta variable se encuentra asociada con bajas competencias y con bajos rendimientos. Con el segundo factor, está asociada con bajos rendimientos pero altas competencias. El resultado de la síntesis de los dos factores indicaría que la repitencia aparece asociada en mayor medida con los bajos rendimientos $y$, aunque en alguna medida se asocia con bajas competencias, esta asociación resulta menos fuerte. En otras palabras, el análisis global muestra que son las evaluaciones de los maestros, en relativa independencia de los niveles de competencia de sus estudiantes, lo que determina la repitencia de los mismos. Otras variables que muestran tendencias contrapuestas son algunas actitudes frente a las asignaturas de lenguaje y ciencias sociales: mejores actitudes muestran mayores rendimientos, pero menores competencias.

Bajo la óptica del estilo cognitivo estos resultados, aparentemente confusos, tienen total sentido. Tal y como se observó del segundo factor, los estudiantes con ciertos niveles de independencia del medio, se ubican en el extremo positivo del segundo factor; esto es, aunque siguen mostrando altas competencias, muestran rendimientos -si se quiere -, "menos buenos" y, al tiempo, presentan alguna repitencia, han sido suspendidos del colegio, se "escapan" del mismo con alguna frecuencia y, en general, han estado en un mayor número de colegios. Lo contrario ocurre con los sensibles, que presentando bajas competencias, son comparativamente mejor evaluados por sus maestros, muestran menos repitencia, son en menor medida castigados con suspensiones, jamás se escapan del colegio y presentan una movilidad escolar menor.

Así, de acuerdo con el examen de estas características a la luz del comportamiento social característico de cada estilo cognitivo, debe concluirse que los maestros consideran reprobables, y así se manifiesta en sus evaluaciones, ciertos comportamientos autónomos más frecuentes en sujetos independientes del medio, sin que esto incida en sus puntajes en pruebas objetivas como la evaluación de competencias. En definitiva, debemos también mantener una segunda hipótesis, aparentemente paradójica si se la compara con la primera: el sistema desfavorece a los sujetos independientes, ya no en el desarrollo de sus competencias, sino en su trayectoria escolar.

Encontramos entonces una contradicción clara $\mathrm{e}$ inherente a nuestro sistema educativo: al tiempo que se favorece a los estudiantes independientes en relación con sus logros objetivos, se les desfavorece en su trayectoria escolar por las características de su comportamiento social. En relación con los estudiantes sensibles al medio, resulta claro que estos no alcanzan los niveles de competencia esperados. Tales estudiantes, sin embargo, avanzan dentro del sistema educativo con fluidez, en buena parte porque su 
característico comportamiento social les permite ajustarse más fácilmente a una situación social jerarquizada y asimétrica como la escolar.

Esto refleja una realidad que no es la mejor de las posibles: tal y como lo hemos planteado reiterativamente, cualquier estudiante, independientemente de su estilo cognitivo, puede lograr las competencias básicas que resultan ser objetivos del sistema educativo. El hecho de que sólo los independientes lo consigan es clara muestra de que no se están dando las oportunidades de desarrollo para el sujeto sensible o bien, de que las pruebas de competencia favorecen la expresión de los logros de los sujetos independientes.

En atención a los resultados, el presente proyecto recomienda la consideración de las particularidades cognitivas de la población colombiana y de las diferencias al interior del salón de clase como condición absolutamente necesaria para el mejoramiento de los niveles de logro de los estudiantes. Para conseguirlo, se proponen dos tipos de acciones, cualitativamente diferentes entre sí, y con efectos seguramente sinérgicos:

- Adoptar, de forma decidida, un enfoque diferencial para la educación colombiana. El efecto de este enfoque es personalizar la prestación del servicio, y elevar los niveles de calidad del sistema. El sistema debe adaptarse a los individuos a los que va dirigido, más que obligar la adaptación de los mismos al esquema preestablecido.

- Propiciar en estudiantes y docentes un mayor desarrollo en las inhabilidades que resultan propias de su estilo cognitivo. Técnicamente, estamos proponiendo una cierta modificación del estilo cognitivo tendiente, entiéndase bien, al logro de una mayor movilidad funcional por parte de sujetos sensibles y de independientes.

Estas dos acciones pueden ser implementadas, en el orden político, a partir de la promoción y la difusión entre los maestros de un enfoque pedagógico de tipo diferencial. La consideración de las diferencias presentes en el interior de clase es condición imprescindible para el logro de todos los estudiantes y particularmente de aquellos que muestran tendencias hacia la sensibilidad. La investigación cognitiva diferencial ha arrojado gran cantidad de información acerca de las necesidades de los sujetos de cada estilo para el logro de sus aprendizajes. Estos resultados deben ser estructurados y difundidos en programas amplios de formación permanente de docentes (PFPD). Puede ser importante para el logro de este propósito mantener grupos escolares pequeños, o por lo menos no demasiado amplios, aunque esta no es una condición suficiente: incluso grupos muy pequeños pueden ser tratados de forma masiva

Además de lo anterior, se recomienda revisar los sistemas de evaluación escolar (la repitencia, en particular, se encontró más asociada con las evaluaciones de los sus maestros que con las pruebas objetivas de competencia) y fomentar actividades de entrenamiento cognitivo de los estudiantes y los docentes (lo cual puede ser logrado de múltiples formas, entre ellas: incrementando, por parte de estudiantes y docentes, el uso de computadores en el colegio e implementando acciones para el desarrollo de competencias sociales y afectivas).

En el orden pedagógico, se recomienda con especial énfasis la necesidad de adoptar un enfoque pedagógico diferencial, lo cual conlleva para el maestro interiorizar el hecho de que, al margen de sus capacidades "reales", los estudiantes presentan estilos cognitivos diferentes y, por lo tanto, requieren otros tipos de información, formas distintas en la presentación de esta información, y diferentes modalidades de evaluación del logro. De igual forma, adoptar este tipo de enfoque señala la importancia de destinar tiempo suficiente para una atención estrecha del maestro a los estudiantes sensibles, diversificar 
los tipos y las fuentes de la información presentada, y diversificar las practicas evaluativas.

En el orden técnico se recomienda avanzar en el perfeccionamiento de los instrumentos para la evaluación de competencias básicas, algunos muestran problemas técnicos en relación con su validez, su especificidad y su poder de discriminación.

\section{Referencias}

ABRAHAM, R.G. (1985) Field independence/dependence and the teaching of grammar. TESOL Quarterly, 20,689-702.

AL NESIR, R.; KEENAN, V. y LANGER, P (1991) Field dependence-independence in the development of referential communication. Bulletin of te Psychonomic or Society, 29, 17-18.

ALPTEKIN, C. y ATAKAN, S. (1990) Field dependence-independence and hemisphericity as variables in L2 achievement. Second Language Research, 6. 135-149.

BARBE, V. y TOURRETTE, C. (1994) Acquisition des marques de position spatiale et dépendence indépendence a l'égard du champ chez des enfants de maternelle. L'Année Psychologique, 95,425-440.

BIALYSTOCK, E. (1992) Attentional control in children's metalinguistic performance and measure of field dependence. Development Psychology, 28(4), 654664.

BURWELL, L. B. (1991) The interactions of learning styles with leamer control treatment in an interactive videodisc lesson. Educational Techology, 31,37-43.

DEPARTAMENTO NACIONAL DE PLANEACIÓN (1999) Un Plan Educativo para la Paz. Santa fe de Bogotá: Departamento Nacional de Planeación.

HEDERICH, C. y CAMARGO, A. (1993) Diferencias Cognitivas y Subculturas en Colombia, Santa fe de Bogotá: Universidad Pedagógica Nacional Colciencias.

HEDERICH, C; CAMARGO, A.; GUZMÁN, L. y PACHECO, J.C. (1995) Regiones Cognitivas en Colombia. Santafe de Bogotá: Universidad Pedagógica Nacional - Colciencias.

HEDERICH, C. y CAMARGO, A. (1995) Logro educativo y estilo cognitivo en Colombia. Revista Colombiana de Educación, 30, 67-86.

HEDERICH, C. y CAMARGO, A. (1999a) Estilos cognitivos como modalidades de procesamiento de la información. Santa fe de Bogotá Universidad Pedagógica Nacional-Colciencias.

HEDERICH, C. y CAMARGO, A. (1999b) Estilos Cognitivos en Colombia. Resultados en cinco regiones culturales. Santa fe de Bogotá: Universidad Pedagógica Nacional -Colciencias.

KUSH, J.P. (1996) Field-dependence, cognitive ability and academic achievement in Anglo-American and Mexican-American students. Journal of CrossCultural Psychology, 27(5), 561-575.

MEN-SABER (1992) Sistema Nacional de Evaluación de la Calidad de la Educación. Primeros Resultados: Matemáticas y Lenguaje en Básica Primaria. Santa fe de Bogotá: Ministerio de Educación Nacional. Colección Saber No. 1.

MORENO, Guadalupe (1998) El desarrollo de habilidades como objetivo educativo. Una aproximación conceptual. Educar. Nueva Era. Julio-Septiembre 1998 SALTERLEY, D.J. (1976) Cognitive styles, spatial ability and school achievement, Journal of Educational Psychology, 68,36-42.

SECRETARIA DE EDUCACIÓN DEL DISTRITO (1999). Guía Evaluación de Competencias Básicas en Lenguaje, Matemática y Ciencias. Octubre de 1999. Bogotá: SED. 
SECRETARIA DE EDUCACIÓN DEL DISTRITO. (1999) Resultados Evaluación de Competencias Básicas en Lenguaje, matemática y Ciencias. Tercera Aplicación. Calendario A, Octubre 1999. Bogotá: SED.

TEDESCO, J.C. (1992) Estrategias de desarrollo y educación. El desafío de la gestión pública. En: Revista Colombiana de Educación No. 24, Bogotá: Universidad Pedagógica Nacional-CIUP, 7-21.

TENNANT, (1988) Psychology and Adult Learning. London: Routledge.

TINAJERO, C. y PÁRAMO, M.F.(1997) Field dependence-independence and academic achievement : a re-examjnation of their relationship. British Journal of Educational Psychology, 67, 199-2 12.

WITKIN, H. y GOODENOUGH, D. (1981) Estilos Cognitivos. Naturaleza y orígenes. Madrid: Ediciones Pirámide. 